Ergod. Th. \& Dynam. Sys. (1986), 6, 571-582

Printed in Great Britain

\title{
The spectra of topological Markov shifts
}

\author{
D. A. LIND \\ Department of Mathematics, University of Washington, Seattle, \\ Washington 98195, USA
}

(Received 25 July 1985 and revised 17 January 1986)

\begin{abstract}
For every Perron number $\lambda$ we construct an infinite collection of topological Markov shifts with entropy $\log \lambda$ whose spectra are disjoint except for the necessary conjugates of $\lambda$. This is used to show that Marcus' theorem about every Markov shift of entropy $\log n$ factoring onto the full $n$-shift does not extend to certain entropy values.
\end{abstract}

\section{Background}

Let $A$ be a square non-negative integral matrix, which we assume throughout to be aperiodic in that some power of $A$ is strictly positive. A familiar procedure [W] associates to such an $A$ a homeomorphism $\sigma_{A}$ of a totally disconnected compact space $X_{A}$. The topological entropy $h\left(\sigma_{A}\right)$ of $\sigma_{A}$ equals $\log \lambda_{A}$, where $\lambda_{A}$ is the largest eigenvalue of $A$. For a detailed treatment of these topological Markov shifts and their central role in dynamics see [DGS].

We say that $\sigma_{A}$ factors onto $\sigma_{B}$ if there is a continuous surjection $\pi: X_{A} \rightarrow X_{B}$ with $\pi \sigma_{A}=\sigma_{B} \pi$. A shift $\sigma_{A}$ is said to be minimal in its entropy class if every shift with the same entropy factors onto it. Marcus [M] has shown that the full $n$-shift is minimal in its entropy class for rational integers $n \geq 2$.

Recently Trow [T] established a beautiful generalization of Marcus' theorem. He showed that the existence of factor maps is closely related to classes of ideals in $\mathbb{Z}[1 / \lambda]$ generated by coordinates of eigenvectors. In particular, if $C \geq 0$ is aperiodic with irreducible characteristic polynomial, and $\mathbb{Z}[1 / \lambda]$ is a principal ideal domain then for every $\sigma_{A}$ with $\lambda_{A}=\lambda_{C}$ there is an $n \geq 1$ such that $\sigma_{A}^{n}$ factors onto $\sigma_{C}^{n}$.

Our purpose is to show that these results cannot generalize directly to arbitrary $\lambda$. We show in theorem 3 that if the trace of $\lambda$ is strictly negative and the other conjugates of $\lambda$ have negative real part, then there is no finite collection $\left\{\sigma_{C_{1}}, \ldots, \sigma_{C_{n}}\right\}$ of shifts with entropy $\log \lambda$ such that every $\sigma_{A}$ with $h\left(\sigma_{A}\right)=\log \lambda$ has some power that factors onto the same power of some $\sigma_{C_{i}}$. We do this by constructing rather explicitly, for every $\lambda$ and sufficiently large $M$, a sequence of aperiodic matrices whose spectra converge to the $M$ th roots of unity times the conjugates of $\lambda$ together with the circle of radius $\lambda$ (theorem 1). Rouché's theorem is used to locate the spectra of these matrices. The geometric localization of the eigenvalues together with Galois theory makes it easy to extract a subsequence of these matrices whose spectra are disjoint except for the necessary conjugates of 
$\lambda$ in common (theorem 2). A result of Kitchens on divisibility of zeta functions then gives the conclusion of theorem 3 .

The exact statements are given in $\S 2$. In $\S 3$ we describe the algorithm from [L] that produces all non-negative integral matrices with prescribed spectral radius. In $\$ 4$ we show how this works for three pivotal examples, which together contain all the significant ideas of the general proof. Even in the simplest case when $\lambda$ is a rational integer, our method yields an interesting family of irreducible polynomials. The reader is urged to study these examples before reading the proofs, which are supplied in $\S 5$.

The main ideas behind the proof of theorem 1 were discovered experimentally using the interactive MATLAB linear algebra package. We thank Roy Adler for suggesting the original problem, and Don Marshall for several useful conversations.

This research was supported in part by NSF Grant DMS-8320356.

\section{Theorems}

Define $\lambda$ to be a Perron number if it is an algebraic integer $\geq 1$ whose conjugates have absolute value $<\lambda$, and denote by $\mathbb{P}$ the set of such numbers. In $[\mathbf{L}]$ it is shown that $\lambda$ is a Perron number iff it is the spectral radius of an aperiodic non-negative integral matrix.

For $\lambda \in \mathbb{P}$ with degree $d$, let $p(t)$ be the minimal polynomial of $\lambda$ over $\mathbb{Q}$. Thus $p(t)=\prod_{j=1}^{d}\left(t-\lambda_{j}\right)$, where $\lambda_{1}=\lambda, \lambda_{2}, \ldots, \lambda_{d}$ are the conjugates of $\lambda$. If $A$ has spectral radius $\lambda_{A}=\lambda$, then the characteristic polynomial $\chi_{A}(t)$ of $A$ has $\lambda$ as a root. Since $p(t), \chi_{A}(t) \in \mathbb{Z}[t]$ and $p(t)$ is irreducible, $p(t)$ divides $\chi_{A}(t)$, and hence each $\lambda_{i}$ is an eigenvalue of $A$. By the Perron-Frobenius theory [G], each is simple.

The first theorem constructs a sequence of matrices whose spectra become geometrically localized. For $\lambda \in \mathbb{P}, M \geq 1$, and $\delta>0$, let $E(\lambda, M, \delta)$ denote the set of complex numbers within $\delta$ of an $M$ th root of unity times a distinct conjugate of $\lambda$, together with the annulus $\{(1-\delta) \lambda<|z| \leq \lambda\}$.

TheOREM 1. Let $\lambda \in \mathbb{P}$. There is an $M_{0}(\lambda)$ such that if $M \geq M_{0}(\lambda)$, there exists $a$ sequence $\left\{A_{n}\right\}$ of aperiodic non-negative integral matrices with $\lambda_{A_{n}}=\lambda$ such that for every $\delta>0$ the eigenvalues of $A_{n}$ are eventually in $E(\lambda, M, \delta)$.

It was noted above that every $A$ with $\lambda_{A}=\lambda$ has $\chi_{A}(t)$ divisible by $p(t)$. However, theorem 1 together with Galois theory allows inductive construction of an infinite collection of such $A$ 's whose characteristic polynomials have only $p(t)$ in common.

THEOREM 2. For every $\lambda \in \mathbb{P}$ there exists a sequence of aperiodic non-negative integral matrices with spectral radius $\lambda$ such that their characteristic polynomials have pairwise only $p(t)$ in common.

The theorems above rule out a direct generalization of the results of Marcus and Trow to certain values of $\lambda$. Let $\Sigma_{\lambda}$ denote the set of mixing topological Markov shifts with entropy $\log \lambda$. 
THEOREM 3. Let $\lambda \in \mathbb{P}$ have negative trace and the other conjugates of $\lambda$ have negative real part. There is no finite collection $\left(\sigma_{C_{1}}, \ldots, \sigma_{C_{n}}\right\} \subset \Sigma_{\lambda}$ such that every $\sigma_{A} \in \Sigma_{\lambda}$ factors onto some $\sigma_{C_{i}}$. Indeed, there is no such collection such that for every $\sigma_{A} \in \Sigma_{\lambda}$ there is an integer $k \geq 1$ so that $\sigma_{A}^{k}$ factors onto some $\sigma_{C_{i}}^{k}$.

The assumption on the conjugates of $\lambda$ is only needed to prove the second statement, and likely are not needed at all. See the remarks following the proof of theorem 3 .

\section{Matrices}

Fix $\lambda \in \mathbb{P}$. We review here the general method from [L] for constructing all aperiodic matrices $A$ over the non-negative integers $\mathbb{Z}^{+}$with $\lambda_{A}=\lambda$.

Let $\operatorname{deg} \lambda=d$, and $B$ be the $d$-dimensional companion matrix of the minimal polynomial $p(t)$ for $\lambda$. Of course $B$ need not be non-negative. Under the action of $B$ there is an invariant splitting of $\mathbb{R}^{d}$ into $C \oplus D$, where $D$ is the one-dimensional dominant eigenspace for $\lambda$, and $C$ is its $B$-invariant complement. Let $\pi_{D}: \mathbb{R}^{d} \rightarrow D$ denote projection to $D$ along $C$. Identifying $D$ with $\mathbb{R}$, we can speak of the $D$-coordinate of $x \in \mathbb{R}^{d}$ as being $\pi_{D}(x)$.

Suppose there is an $n$-tuple $\left(z_{1}, \ldots, z_{n}\right)$ of integral vectors in $\mathbb{R}^{d}$ with $\pi_{D}\left(z_{i}\right)>0$ for $1 \leq i \leq n$, and such that for each $j$,

$$
B z_{j}=\sum_{i=1}^{n} a_{i j} z_{i}, \quad a_{i j} \in \mathbb{Z}^{+} .
$$

Form the $n$-dimensional matrix $A=\left[a_{i j}\right]$. The argument at the end of the proof of theorem 1 in $[\mathbf{L}]$ shows that $\lambda_{A}=\lambda$, although $A$ may not be aperiodic or even irreducible. Conversely, if $A=\left[a_{i j}\right]$ is an arbitrary aperiodic matrix over $\mathbb{Z}^{+}$with $\lambda_{A}=\lambda$, by $\left[\mathbf{L}\right.$, theorem 2] there is an $n$-tuple $\left(z_{1}, \ldots, z_{n}\right)$ of integral vectors in $\mathbb{Z}^{d}$ with $\pi_{D} z_{i}>0$ such that (3.1) holds. The latter is proved by using a $n$-dimensional right eigenvector for $\lambda$ under $A$ whose coordinates lie in $\mathbb{Z}[\lambda]$, and identifying elements of $\mathbb{Z}[\lambda]$ with integral vectors in $\mathbb{Z}^{d}$.

In the next two sections, we will describe one systematic way of constructing, for all large enough $n, n$-tuples of integral vectors satisfying the above conditions. We will then analyze the corresponding sequence of matrices and their spectra to prove theorem 1.

\section{Examples}

We shall illustrate the main ideas in the proofs of theorems 1 and 2 by showing how they work on three key examples. We call these the linear, cubic, and quadratic examples. The first, when $\lambda$ is a rational integer, shows how geometric localization and separation of the spectra work. Even in this simple case, the method yields an interesting sequence of irreducible polynomials. Next we treat a particular cubic algebraic integer $\lambda$ with negative trace. This provides a concrete example for theorem 3 and its proof. Special features of this cubic $\lambda$ are used in the proof to obtain $M_{0}(\lambda)=1$. The possibility that $M_{0}(\lambda)$ is forced to be larger arises in the last, quadratic example. Essentially the entire proof is contained here in simplified form. 
(i) A linear example. Consider $\lambda=K \geq 2$. Here $d=1$ and $B=[K]$. Let $z_{1}=1$, $z_{2}=B z_{1}, \ldots, z_{N}=B z_{N-1}=K^{N-1}$. The simplest way to guarantee that an irreducible matrix $A$ is aperiodic is for $\operatorname{tr} A>0$. Thus in (3.1) we use

together with

$$
B z_{N}=K^{N}=(K-1) K^{N-1} z_{1}+z_{N}
$$

$$
B z_{j}=z_{j+1} \quad \text { for } 1 \leq j \leq N-1 .
$$

This produces an aperiodic matrix $A_{N}$ that is the companion matrix of

$$
t^{N}-t^{N-1}-(K-1) K^{N-1}=\chi_{A_{N}}(t)=\chi_{N}(t) .
$$

Let $\delta>0$. We show that for sufficiently large $N$ all roots $\mu$ of $\chi_{N}$ obey $(1-\delta) K<$ $|\mu| \leq K$, i.e. they lie in $E(K, 1, \delta)$. Since $A$ is aperiodic with $\lambda_{A}=K$, the PerronFrobenius theorem shows $|\mu| \leq K$. If $\chi_{N}(\mu)=0$, then

so that

$$
\mu^{N-1}(\mu-1)=(K-1) K^{N-1},
$$

$$
|\mu|=\left(\frac{K-1}{|\mu-1|}\right)^{1 /(N-1)} K \geq\left(\frac{K-1}{K+1)}\right)^{1 /(N-1)} K>(1-\delta) K,
$$

for sufficiently large $N$.

A variant of this argument, employing Rouché's theorem, extends to the general case. We give it here for comparison. Let $f_{N}(t)=t^{N}-(K-1) K^{N-1}$ and $g_{N}(t)=t^{N-1}$. If $|t| \leq \rho=K((K-1) / 2 K)^{1 /(N-1)}$, then

$$
\left|t^{N}\right| \leq K^{N}\left(\frac{K-1}{2 K}\right)^{N /(N-1)}<K^{N}\left(\frac{K-1}{2 K}\right)=\frac{1}{2}(K-1) K^{N-1},
$$

so $\left|f_{N}(t)\right|>\frac{1}{2}(K-1) K^{N-1}$ for $|t| \leq \rho$. If $|t|=\rho$ then

$$
\left|g_{N}(t)\right|=\rho^{N-1}=\frac{1}{2 K}(K-1) K^{N-1}<\left|f_{N}(t)\right| .
$$

By Rouché's theorem, since $f_{N}(t)$ has no zeros in $\{|t| \leq \rho\}$, neither does $f_{N}(t)-g_{N}(t)=\chi_{N}(t)$. Thus all roots of $\chi_{N}$ lie in $\{\rho<|t| \leq K\}$. Since $\rho \nearrow K$ as $N \rightarrow \infty$, this proves theorem 1 in the linear case.

A simple inductive construction now proves theorem 2. Denote the spectrum of $A$ by $\operatorname{sp}(A)$. Pick $\delta_{1}>0$ and by the above choose $N_{1}$ so that $\left(1-\delta_{1}\right) K<|\mu| \leq K$ for $\mu \in \operatorname{sp}\left(A_{N_{1}}\right)$. Pick $\delta_{2}>0$ so that $\delta_{2} \leq \frac{1}{2} \delta_{1}$ and

$$
\max \left\{|\mu|: \mu \in \operatorname{sp}\left(A_{1}\right), \mu \neq K\right\}<\left(1-\delta_{2}\right) K,
$$

and produce $A_{N_{2}}$ with sp $\left(A_{N_{2}}\right) \subset\left\{\left(1-\delta_{2}\right) K<|t| \leq K\right\}$. Continuing, we obtain $A_{N_{n}}$ with $\operatorname{sp}\left(A_{N_{i}}\right) \cap \operatorname{sp}\left(A_{N_{j}}\right)=\{K\}$, so their characteristic polynomials have only $p(t)=$ $t-K$ in common.

It is possible to estimate the growth of $\operatorname{dim} A_{N_{n}}$ as follows. The argument above shows there is a constant $c_{1}$ so that

$$
\left(1-\frac{c_{1}}{N}\right) K<|\mu|, \quad \mu \in \operatorname{sp}\left(A_{N}\right)
$$


A careful analysis of the positions of the elements in $\operatorname{sp}\left(A_{N}\right)$ shows there is a $c_{2}$ so that

$$
|\mu|<\left(1-\frac{c_{2}}{N^{3}}\right) K, \quad \mu \in \operatorname{sp}\left(A_{N}\right), \mu \neq K .
$$

It follows that the $N_{n}$ can be chosen so that $N_{n+1} \leq\left(c_{1} / c_{2}\right) N_{n}^{3}$, and hence that $\operatorname{dim} A_{N_{n}}=O\left(\exp \left(\exp \left(c_{3} n\right)\right)\right)$ for suitable $c_{3}$.

For the linear case the characteristic polynomials produced are in fact irreducible modulo $p(t)=t-K$. This fact, and its proof, were pointed out to us by David Boyd.

Proposition 1. With the above notation, the polynomials

$$
\frac{\chi_{N}(t)}{t-K}=\frac{t^{N}-t^{N-1}-(K-1) K^{N-1}}{t-K}
$$

are irreducible for $N, K \geq 2$.

Proof. Let $q(t)=\chi_{N}(t) /(t-K)$, and put

$$
r(t)=\frac{1}{K^{N-2}} q(K t)=K t^{N-1}+(K-1)\left(t^{N-2}+\cdots+1\right) .
$$

All roots $\mu$ of $r(t)$ have $|\mu|<1$. It suffices to show $r(t)$ is irreducible over $\mathbb{Z}[t]$. Suppose to the contrary that $r(t)=\left(a_{0} t^{m}+\cdots+a_{1}\right)\left(b_{0} t^{N-1-m}+\cdots+b_{1}\right)$ with $a_{0}>0$, so $a_{0} b_{0}=K, a_{1} b_{1}=K-1$. We claim $\max \left\{\left|a_{1}\right| / a_{0},\left|b_{1}\right| / b_{0}\right\} \geq 1$. If not, then

$$
\frac{K-1}{K}=\frac{\left|a_{1}\right|}{a_{0}} \frac{\left|b_{1}\right|}{b_{0}} \leq \frac{a_{0}-1}{a_{0}} \frac{b_{0}-1}{b_{0}}=1-\frac{1}{a_{0}}-\frac{1}{b_{0}}+\frac{1}{K},
$$

so $1 / a_{0}+1 / b_{0} \leq 2 / K$. Multiplying by $K$ shows $a_{0}+b_{0} \leq 2$, so $a_{0}=b_{0}=1$, contradicting $a_{0} b_{0}=K$. If now $\left|a_{1}\right| / a_{0} \geq 1$, then the product of the roots of the first factor of $r(t)$ has modulus $\left|a_{1}\right| / a_{0} \geq 1$, contradicting the fact that all roots of $r(t)$ have modulus $<1$. The case $\left|b_{1}\right| / b_{0} \geq 1$ is similar.

(ii) A cubic example. Let $\lambda \approx 3.8916$ be the largest root of $p(t)=t^{3}+3 t^{2}-15 t-46$, which is irreducible. Then $\lambda$ has conjugates $\lambda_{2} \approx-3.2141$ and $\lambda_{3} \approx-3.6775$, so $\lambda \in \mathbb{P}$ and $\operatorname{tr} \lambda=-3$. The companion matrix $B$ for $p(t)$ has a positive eigenvector $w$ for $\lambda$. Let $D=\mathbb{R} w$ and $C$ be the $B$-invariant complement. Recall that $\pi_{D}$ is projection to $D$ along $C$.

We pick integral vectors by letting $e=\left[\begin{array}{lll}1 & 0 & 0\end{array}\right]^{T}$ and $z_{j}=B^{j-1} e(1 \leq j \leq N)$. Now $\pi_{D} e=\alpha w$, where $\alpha>0$ and $w>0$. Since $\left(\lambda^{-1} B\right)^{N} \rightarrow \pi_{D}$, it follows that $B^{N} e>0$ eventually. Surprisingly, $B^{N} e>0$ only for $N \geq 49$. Now $\left\{z_{1}, z_{2}, z_{3}\right\}$ is the standard basis for $\mathbb{R}^{3}$. This allows the following choice of coefficients in (3.1):

$$
\begin{aligned}
B z_{j} & =z_{j+1}, \quad(1 \leq j \leq N-1), \\
B z_{N}=B^{N} e & =\left(B^{N} e\right)_{1} z_{1}+\left(B^{N} e\right)_{2} z_{2}+\left(B^{N} e\right)_{3} z_{3} .
\end{aligned}
$$

This yields the matrix $A_{N}$ that is the companion matrix of

$$
\chi_{N}(t)=t^{N}-\left[\begin{array}{lll}
1 & t & t^{2}
\end{array}\right] B^{N} e .
$$

Since $B^{N} e>0$ for $N \geq 49$, the corresponding $A_{N}$ is both non-negative and aperiodic. 
We shall show that except for $\lambda_{2}$ and $\lambda_{3}$, all roots of $\chi_{N}$ have modulus close to $\lambda$. Normalize $\chi_{N}$ by putting $s=t / \lambda$ and

$$
f_{N}(s)=\lambda^{-N} \chi_{N}(\lambda s)=s^{N}-\left[\begin{array}{lll}
1 & \lambda s & \lambda^{2} s^{2}
\end{array}\right]\left(\lambda^{-N} B^{N} e\right) .
$$

By the Perron-Frobenius theorem, 1 is a root of $f_{N}$ and all other roots lie in $\{|s|<1\}$. Also note that if $\rho$ is fixed with $\left|\lambda_{3}\right| / \lambda<\rho<1$, then since $\pi_{D} e=\alpha w$,

$$
\left\|\lambda^{-N} B^{N} e-\alpha w\right\|<\rho^{N}
$$

for large enough $N$.

Consider $g(s)=\left[\begin{array}{lll}1 & \lambda s & \lambda^{2} s^{2}\end{array}\right] \alpha w$. Now $B$ has left eigenvectors $\left[\begin{array}{lll}1 & \lambda_{i} & \lambda_{i}^{2}\end{array}\right]$ corresponding to $\lambda_{i}(i=2,3)$, and right eigenvector $w$ corresponding to $\lambda$. Orthogonality of eigenvectors belonging to distinct eigenvalues shows $\lambda_{2} / \lambda$ and $\lambda_{3} / \lambda$ are the roots of $g(s)$. Hence $\min \{\mid g(s): \rho \leq s \leq 1\}=\theta>0$.

Let $r=(\theta / 2)^{1 / N}$. We suppose $N$ large enough so $r>\rho$. For $|s|=r$,

$$
\begin{aligned}
& \left|f_{N}(s)-g(s)\right|=\left|s^{N}-\left[\begin{array}{lll}
1 & \lambda s & \lambda^{2} s^{2}
\end{array}\right]\left(\lambda^{-N} B^{N} e-\alpha w\right)\right| \\
& \leq r^{N}+3 \lambda^{2} \rho^{N}<\theta \leq|g(s)|,
\end{aligned}
$$

for $N$ sufficiently large. By Rouché's theorem, $g(s)$ and $f_{N}(s)$ have the same number of zeros in $\{|s| \leq r\}$, namely two. Thus $f_{N}(s)$ has the roots $\lambda_{2} / \lambda, \lambda_{3} / \lambda$ in $\{|s| \leq r\}$, and its other $N-2$ roots in $\{r<|s| \leq 1\}$.

Consequently $\chi_{N}(t)$ has roots $\lambda_{2}$ and $\lambda_{3}$, and its other roots lie in

$$
\left\{(\theta / 2)^{1 / N} \lambda<|t| \leq \lambda\right\} .
$$

If $\delta>0$, then this shows that for $N$ large enough, $\operatorname{sp}\left(A_{N}\right) \subset E(\lambda, 1, \delta)$, proving theorem 1 in this case.

Noting that the roots $\lambda_{i}$ of $\chi_{N}$ are always simple by Perron-Frobenius, the inductive construction for theorem 2 follows as in the linear case.

(iii) $A$ quadratic example. The key feature leading to the simplicity of the cubic example was the existence of an integral vector $e$ so that the dominant eigendirection was in the positive cone of $\left\{e, B e, B^{2} e\right\}$. This feature is not true for the quadratic example below. The proof for this case is more difficult, and shows how roots of unity times conjugates of $\lambda$ arise.

Let

$$
B=\left[\begin{array}{ll}
1 & 1 \\
1 & 2
\end{array}\right],
$$

with eigenvalues $\lambda=(3+\sqrt{5}) / 2 \in \mathbb{P}$ and $\lambda_{2}=(3-\sqrt{5}) / 2$. Let $\alpha=(1+\sqrt{5}) / 2$ and $\beta=(1-\sqrt{5}) / 2$, so $\lambda=\alpha^{2}, \lambda_{2}=\beta^{2}$. The eigenvectors for $B$ are $w_{1}=\left[\begin{array}{ll}1 & \alpha\end{array}\right]^{T}$, $w_{2}=\left[\begin{array}{ll}-\alpha & 1\end{array}\right]^{T}$, so $D=\mathbb{R} w_{1}, C=\mathbb{R} w_{2}$.

Denote by $\pi_{C}$ projection to $C$ along $D$. Since $\lambda_{2}>0$, it follows that for every non-zero $z \in \mathbb{Z}^{2}, 0$ is not a positive combination of $\pi_{C}(z), \pi_{C}(B z), \ldots, \pi_{C}\left(B^{k} z\right)$ for any $k$. Thus $D$ is never contained in the positive cone of $z, B z, \ldots, B^{k} z$.

Let $M$ and $N$ be integers (later we will fix $M$ and let $N \rightarrow \infty$ ). If $\left\{e_{1}, e_{2}\right\}$ is the standard basis, let

$$
\begin{aligned}
z_{j} & =B^{j-1} e_{1} & & (1 \leq j \leq M), \\
z_{M+j} & =B^{j-1} e_{2} & & (1 \leq j \leq N) .
\end{aligned}
$$


Now

$$
B^{n}=\left[\begin{array}{cc}
F_{2 n-1} & F_{2 n} \\
F_{2 n} & F_{2 n+1}
\end{array}\right],
$$

where $F_{n}=\left(\alpha^{n}-\beta^{n}\right) / \sqrt{5}$ is the $n$th Fibonacci number. For the choice of coefficients in (3.1) we use

$$
\begin{aligned}
B z_{j} & =z_{j+1} \quad(1 \leq j \leq M-1, M+1 \leq j \leq M+N-1), \\
B z_{M} & =B^{M} e_{1}=F_{2 M-1} z_{1}+F_{2 M^{2}} z_{M+1}, \\
B z_{M+N} & =B^{N} e_{2}=F_{2 N} z_{1}+F_{2 N+1} z_{M+1} .
\end{aligned}
$$

This produces $A_{M, N}$, which will be aperiodic provided $(M, N)=1$. Row eliminations show that

$$
\chi_{M, N}(t)=\operatorname{det}\left(t I-A_{M, N}\right)=\operatorname{det}\left[\begin{array}{cc}
t^{M}-F_{2 M-1} & -F_{2 N} \\
-F_{2 M} & t^{N}-F_{2 N+1}
\end{array}\right]
$$

Normalize by putting $s=t / \lambda$ and

$$
f_{M, N}(s)=\lambda^{-M-N} \chi_{M, N}(\lambda s)=\operatorname{det}\left[\begin{array}{cc}
s^{M}-\lambda^{-M} F_{2 M-1} & -\lambda^{-N} F_{2 N} \\
-\lambda^{-M} F_{2 M} & s^{N}-\lambda^{-N} F_{2 N+1}
\end{array}\right] \text {. }
$$

Let $U_{M}=\left\{e^{2 \pi i j / M}: 0 \leq j \leq M-1\right\}$. We will find $M_{0}(\lambda)$ below so that if $M \geq M_{0}(\lambda)$, then for $\delta>0$ and all sufficiently large $N, f_{M, N}(s)$ will have $M$ roots within $\delta$ of $\left(\lambda_{2} / \lambda\right) U_{M}$, and the other $N$ roots in $\{1-\delta<|s| \leq 1\}$. This will show that $\operatorname{sp}\left(A_{M, N}\right) \subset E(\lambda, M, \delta)$ and establish theorem 1 here.

Let

$$
g_{M, N}(s)=\operatorname{det}\left[\begin{array}{cc}
s^{M}-1 / \alpha \sqrt{5} & -1 / \sqrt{5} \\
-1 / \sqrt{5} & s^{N}-\alpha / \sqrt{5}
\end{array}\right]=s^{M}\left(s^{N}-\xi s^{N-M}-\eta\right),
$$

where $\xi, \eta>0$ are absolute constants. Clearly 0 is a root of multiplicity $M$ for $g_{M, N}$. Let $\mu \neq 0$ be another. Since $\xi+\eta=1$, it follows $|\mu| \leq 1$. Then

$$
\mu^{N-M}\left(\mu^{M}-\xi\right)=\eta
$$

and since $|\mu| \leq 1$,

$$
|\mu| \geq\left(\frac{\eta}{1+\xi}\right)^{1 /(N-M)}>1-\delta
$$

for $N-M$ large enough.

Put $\rho=\lambda_{2} / \lambda<1$ and fix $r \in(\rho, 1)$. Then $\lambda^{-n} F_{2 n}=(1 / \sqrt{5})+\mathcal{O}\left(\rho^{n}\right)$, so multilinearity of det shows there is an absolute constant $\kappa$ so that for $N \geq M,|s| \leq 1$,

$$
\left|f_{M, N}(s)-g_{M, N}(s)\right| \leq \kappa \rho^{M} \text {. }
$$

For $|s|=r$ we have

$$
\left|g_{M, N}(s)\right| \geq r^{M}\left(\eta-\xi r^{N-M}-r^{N}\right)>\frac{\eta}{2} r^{M}
$$

for $N$ and $N-M$ sufficiently large. Fix $M_{0}(\lambda)$ so that if $M \geq M_{0}(\lambda)$ then $\kappa \rho^{M}<$ $\frac{1}{2} \eta r^{M}$. Fix $M \geq M_{0}(\lambda)$ and let $0<\delta<1-r$. Then from (4.1) and (4.2) we conclude by Rouché's theorem that $f_{M, N}$ and $g_{M, N}$ have an equal number of zeros in $\{|s| \leq r\}$, namely $M$. 
Let $\mu$ be a root of $f_{M, N}$ with $|\mu|>r$. Then (4.1) shows

$$
\left|\mu^{M}\left(\mu^{N}-\xi \mu^{N-M}-\eta\right)\right| \leq \kappa \rho^{M},
$$

so

$$
\left|\mu^{N}-\xi \mu^{N-M}-\eta\right| \leq \kappa\left(\frac{\rho}{r}\right)^{M}<\frac{\eta}{2},
$$

and hence as before

$$
|\mu|>\left(\frac{\eta}{2(1+\xi)}\right)^{1 /(N-M)}>1-\delta,
$$

for $N-M$ sufficiently large.

It remains to locate the $M$ roots of $f_{M, N}$ in $\{|s| \leq r\}$. As $N \rightarrow \infty$ note that $f_{M, N} \rightarrow f_{M, \infty}$ uniformly on $\{|s| \leq r\}$, where

$$
f_{M, \infty}(s)=\operatorname{det}\left[\begin{array}{cc}
s^{M}-\lambda^{-M} F_{2 M-1} & -1 / \sqrt{5} \\
-\lambda^{-M} F_{2 M} & -\alpha / \sqrt{5}
\end{array}\right] .
$$

A calculation shows $f_{M, \infty}(s)=(-\alpha / \sqrt{5})\left[s^{M}-\left(\lambda_{2} / \lambda\right)^{M}\right]$. The fact that the roots of $f_{M, \infty}$ are $\left(\lambda_{2} / \lambda\right) U_{M}$ can also be seen by changing bases from $\left\{e_{1}, e_{2}\right\}$ to $\left\{\pi_{C} e_{1}, \pi_{D} e_{2}\right\}$. Under this change the determinant (4.3) becomes

$$
\operatorname{det}\left[\begin{array}{cc}
\chi\left(s^{M}\right) & 0 \\
* & 1
\end{array}\right]
$$

where $\chi(s)$ is the characteristic polynomial of the restriction to $C$ of $\lambda^{-M} B^{M}$. A standard application of Rouché's theorem now shows that for sufficiently large $N, f_{M, N}(s)$ has exactly one root within $\delta$ of each $\left(\lambda_{2} / \lambda\right) \omega, \omega \in U_{M}$. This completes the analysis of $\operatorname{sp}\left(\boldsymbol{A}_{M, N}\right)$.

To prove theorem 2 for this example, fix $M \geq M_{0}(\lambda)$, and choose $N_{n} \rightarrow \infty$ inductively as before, with the added constraint $\left(M, N_{n}\right)=1$. The induction can continue provided that $\operatorname{sp}\left(A_{M, N}\right)$ contains no $\omega \lambda_{2}$ with $1 \neq \omega \in U_{M}$. The validity of this is contained in the following result.

Proposition 2. Let $A$ be an aperiodic matrix over $\mathbb{Z}^{+}$with $\lambda_{A}=\lambda$. Suppose that $\omega$ is a root of unity, and that $\mu$ is a conjugate of $\lambda$ such that $\omega \mu$ is an eigenvalue of $A$. Then $\omega=1$.

Proof. Let $K$ be the splitting field of $\chi_{A}$ over $\mathbb{Q}$, so $\mathbb{Q} \subset \mathbb{Q}(\lambda) \subset K$. The mapping $\tau: \mu \rightarrow \lambda$ extends to an element $\tau$ of the Galois group of $K / \mathbb{Q}$. Now $\tau(\omega)$ is a root of unity, so $\tau(\omega \mu)=\tau(\omega) \lambda$ is an eigenvalue of $A$ with modulus $\lambda$. By the PerronFrobenius theorem, $\tau(\omega) \lambda=\lambda$, proving $\tau(\omega)=1$, so $\omega=1$.

\section{Proofs}

We have now laid the preparations for the proof of theorems 1-3.

Proof of theorem 1. Fix $\lambda \in \mathbb{P}$, and let the notation be as in $\S 3$. The proof when $\operatorname{deg} \lambda=1$ is given in $\S 4$ (i), so assume $\operatorname{deg} \lambda \geq 2$. We begin by finding an integral basis for $\mathbb{Z}^{d}$ whose positive cone contains the dominant eigenvector $w$ for $B$.

LEMma 1. (Handelman $[\mathrm{H}])$. There are $u_{1}, \ldots, u_{d} \in \mathbb{Z}^{d}$ forming an integral basis for $\mathbb{Z}^{d}$ such that $\pi_{D} u_{j}>0$ for $1 \leq j \leq d$, and $w$ is a strictly positive combination of the $u_{j}$. 
Proof. Let $U=\left[u_{1} \cdots u_{d}\right] \in G L(d, \mathbb{Z})$, and $w^{*}$ be a left eigenvector for $B$ belonging to $\lambda$ such that $\pi_{D} x=w^{*} x$. The conditions $\pi_{D} u_{j}>0$ are equivalent to $w^{*} U>0$. The other condition is that there be a $c>0$ such that $w=U c$, or $U^{-1} w>0$. The existence of $U \in G L(d, \mathbb{Z})$ simultaneously satisfying $w^{*} U>0$ and $U^{-1} w>0$ is established in the proof of theorem $I$ in $[\mathbf{H}]$.

Now fix $\rho$ and $r$ with

$$
\max _{2 \leq j \leq d} \frac{\left|\lambda_{j}\right|}{\lambda}<\rho<r \leq R=r^{1 /(d-1)}<1 .
$$

LeMMA 2. $\left\|\lambda^{-n} B^{n}-\pi_{D}\right\|=O\left(\rho^{n}\right)$ as $n \rightarrow \infty$.

Proof. Since $\lambda^{-1} B$ has a simple eigenvalue of 1 with eigenvector $w$, and its other eigenvalues are $\lambda_{j} / \lambda(2 \leq j \leq d)$, this follows since $\left|\lambda_{j} / \lambda\right|<\rho$.

LEMMA 3. There is an $M_{1}(\lambda)$ such that if $M \geq M_{1}(\lambda)$, then each $B^{M} u_{j}$ is a strictly positive combination of $u_{1}, \ldots, u_{d}$.

Proof. Letting $U=\left[u_{1} \cdots u_{d}\right]$, by lemma $1, U^{-1} w>0$. By lemma $2, \lambda^{-M} B^{M} \rightarrow \pi_{D}$, so $\lambda^{-M} B^{M} u_{j} \rightarrow \pi_{D}\left(u_{j}\right) w$, where $\pi_{D}\left(u_{j}\right)>0$ by lemma 1 . Hence

$$
U^{-1}\left(\lambda^{-M} B^{M} u_{j}\right) \rightarrow \pi_{. D}\left(u_{j}\right) U^{-1} w>0,
$$

proving that for each $j, B^{M} u_{j}$ is eventually a strictly positive combination of the $u_{i}$.

Fix $M \geq M_{1}(\lambda)$ and $N \geq M$, and construct $A_{M, N}$ using $\S 3$ as follows. Let $z_{i j}=B^{j-1} u_{i}$ for $1 \leq i \leq d-1,1 \leq j \leq M$, and also $i=d, 1 \leq j \leq N$. Since $M, N \geq M_{1}(\lambda)$, there are $\gamma_{k i}>0$ such that

$$
\begin{aligned}
& B z_{i M}=B^{M} u_{i}=\sum_{k=1}^{d} \gamma_{k i} u_{k}, \quad(1 \leq i \leq d-1), \\
& B z_{d N}=B^{N} u_{d}=\sum_{k=1}^{d} \gamma_{k d} u_{k} .
\end{aligned}
$$

These relations together with $B z_{i j}=B^{j} u_{i}=z_{i, j+1}$ for the remaining $z_{i j}$ define a matrix $A_{M, N}$ of size $(d-1) M+N$ which will be aperiodic if $(M, N)=1$.

Let $G=\left[\gamma_{i j}\right]$ and put $\Delta(t)=\operatorname{diag}\left(t^{M}, \ldots, t^{M}, t^{N}\right)$. Using row eliminations, the determinant defining the characteristic polynomial $\chi_{M, N}(t)$ of $A_{M, N}$ can be reduced to the $d$-dimensional

$$
\chi_{M, N}(t)=\operatorname{det}[\Delta(t)-G] .
$$

The columns of $G$ are just coordinates of $B^{M} u_{i}$ or $B^{N} u_{d}$ with respect to the basis $\left\{u_{1}, \ldots, u_{d}\right\}$, so changing to this basis gives

$$
\chi_{M, N}(t)=\operatorname{det}\left[\left(t^{M} I-B^{M}\right) u_{1}, \ldots,\left(t^{M} I-B^{M}\right) u_{d-1},\left(t^{N} I-B^{N}\right) u_{d}\right] .
$$

Normalize by setting $s=t / \lambda$ and

$$
\begin{aligned}
f_{M, N}(s) & =\lambda^{-(d-1) M-N} \chi_{M, N}(s) \\
& =\operatorname{det}\left[\left(s^{M} I-\lambda^{-M} B^{M}\right) u_{1}, \ldots,\left(s^{N} I-\lambda^{-N} B^{N}\right) u_{d}\right] .
\end{aligned}
$$

By lemma $2, \lambda^{-M} B^{M} u_{j}=\alpha_{j} w+O\left(\rho^{M}\right)$, where $\alpha_{j}=\pi_{D} u_{j}>0$. Hence if

$$
g_{M, N}(s)=\operatorname{det}\left[s^{M} u_{1}-\alpha_{1} w, \ldots, s^{N} u_{d}-\alpha_{d} w\right],
$$


expansion of $f_{M, N}(s)$ by multilinearity shows

$$
f_{M, N}(s)=g_{M, N}(s)+\rho^{M} h(s),
$$

where $h(s)$ is a sum of at most $d$ ! monomials with coefficients uniformly bounded by an absolute constant $K$.

Expand $g_{M, N}(s)$ by multilinearity to obtain

$$
g_{M, N}(s)=s^{(d-1) M+N}-\xi s^{(d-2) M+N}-\eta s^{(d-1) M},
$$

where $\xi, \eta>0$ depend only on the $\alpha_{i}$ and $w$, hence only on $\lambda$. We first locate the zeros of $g_{M, N}$. Clearly 0 is a zero of multiplicity $(d-1) M$. Suppose $\mu \neq 0$ is another. Now

$$
\xi+\eta=\operatorname{tr}\left[\alpha_{1} w, \ldots, \alpha_{d} w\right]=\operatorname{tr} \pi_{D}=1,
$$

so $|\mu| \leq 1$. Since $\mu^{N}-\xi \mu^{N-M}=\eta$,

$$
|\mu|^{N-M}=\frac{\eta}{\left|\mu^{M}-\xi\right|} \geq \frac{\eta}{1+\xi}
$$

so

$$
r^{1 /(d-1)}=R<\left(\frac{\eta}{1+\xi}\right)^{1 /(N-M)} \leq|\mu| \leq 1,
$$

if $N-M$ is large enough.

We now estimate $g_{M, N}(s)$ on $|s|=R$. Clearly

$$
\left|g_{M, N}(s)\right| \geq R^{(d-1) M}\left(\eta-\xi R^{N-M}-R^{N}\right) \geq \frac{\eta}{4} r^{M},
$$

provided $\xi R^{N-M}, R^{N}<\eta / 4$. Furthermore, since $|h(s)| \leq K d$ ! on $\{|s| \leq 1\}$, we have

$$
\left|f_{M, N}(s)-g_{M, N}(s)\right|=\rho^{M}|h(s)| \leq K d ! \rho^{M} .
$$

Let $M_{2}(\lambda)$ be large enough so $M \geq M_{2}(\lambda)$ implies $K d ! \rho^{M}<(\eta / 4) r^{M}$. Then for $M \geq M_{2}(\lambda)$ and large enough $N-M$, by Rouché's theorem $f_{M, N}(s)$ and $g_{M, N}(s)$ have the same number of roots inside $\{|s| \leq R\}$, namely $(d-1) M$.

Suppose $\mu$ is one of the remaining roots of $f_{M, N}(s)$ with $|\mu|>R$. Then

$$
\left(\mu^{N}-\xi \mu^{N-M}-\eta\right) \mu^{(d-1) M}=\rho^{M} h(\mu),
$$

so since $M \geq M_{2}(\lambda)$,

$$
\left|\mu^{N}-\xi \mu^{N-M}-\eta\right| \leq \frac{\rho^{M}}{R^{(d-1) M}} K d !<\frac{\eta}{4} .
$$

It follows that

$$
|\mu|>\left(\frac{\eta}{2(1+\xi)}\right)^{1 /(N-M)}>1-\delta,
$$

provided only that $N-M$ is large enough.

We conclude the proof by locating the $(d-1) M$ roots of $f_{M, N}(s)$ inside $\left\{|s| \leq r_{1}\right\}$. There, as $N \rightarrow \infty, f_{M, N}(s) \rightarrow f_{M, \infty}(s)$ uniformly, where

$$
f_{M, \infty}(s)=\operatorname{det}\left[\left(s^{M}-\lambda^{-M} B^{M}\right) u_{1}, \ldots,\left(s^{M}-\lambda^{-M} B^{M}\right) u_{d-1}, \alpha_{d} w\right] \text {. }
$$


Using the basis $\left\{\pi_{C} u_{1}, \ldots, \pi_{C} u_{d-1}, \pi_{D} w\right\}$, this determinant becomes

$$
f_{M, \infty}(s)=\operatorname{det}\left[\begin{array}{cc}
\chi\left(s^{M}\right) & 0 \\
* & 1
\end{array}\right]=\chi\left(s^{M}\right),
$$

where $\chi(s)$ is the characteristic polynomial of the restriction to $C$ of $\lambda^{-M} B^{M}$, with roots $\left(\lambda_{j} / \lambda\right)^{M}(2 \leq j \leq d)$. Therefore $f_{M, \infty}(s)$ has roots $\omega \lambda_{j} / \lambda\left(2 \leq j \leq d, \omega \in U_{M}\right)$. A standard application of Rouchés theorem then shows that for all large enough $N$, each root of $f_{M, N}(s)$ lies within $\delta$ of one of the $\omega \lambda_{j} / \lambda$.

Letting $M_{0}(\lambda)=\max \left\{M_{1}(\lambda), M_{2}(\lambda)\right\}$, the above shows that given $M \geq M_{0}(\lambda)$ and $\delta>0$, for $N$ sufficiently large and relatively prime to $M$ we have

$$
\operatorname{sp}\left(A_{M, N}\right) \subset E(\lambda, M, \delta),
$$

concluding the proof.

Proof of theorem 2. Pick $M \geq M_{0}(\lambda)$, and choose $N_{n}$ inductively as follows. Pick $\delta_{1}>0$ and using theorem 1 find $N_{1}$ with $\left(M, N_{1}\right)=1$ and $\operatorname{sp}\left(A_{M, N_{1}}\right) \subset E\left(\lambda, M, \delta_{1}\right)$. By proposition 2 of $\S 4$, there is a $\delta_{2}>0$ such that

$$
\operatorname{sp}\left(A_{M, N_{1}}\right) \cap E\left(\lambda, M, \delta_{2}\right)=\left\{\lambda_{1}, \ldots, \lambda_{d}\right\} .
$$

Find $N_{2}$ with $\left(M, N_{2}\right)=1$ and $\operatorname{sp}\left(A_{M, N_{2}}\right) \subset E\left(\lambda, M, \delta_{2}\right)$. Continuing gives $N_{n}$ with $A_{M, N_{n}}$ having the required properties.

Proof of theorem 3. Let $\lambda \in \mathbb{P}$ with $\operatorname{tr} \lambda<0$ and $\operatorname{Re} \lambda_{j}<0$ for $2 \leq j \leq d$. Let $z \in \mathbb{Z}^{d}$ have $\pi_{D}(z)>0$. We claim that the eigenvector $w$ for $\lambda$ is in the strictly positive cone of $\left\{z, B z, \ldots, B^{d-1} z\right\}$. Let $T=\left.B\right|_{C}$ and $x=\pi_{C}(z) \neq 0$. Applying $\pi_{C}$, our claim is equivalent to 0 being a positive combination of $x, T x, \ldots, T^{d-1} x$. Now $\chi_{T}(t)=\prod_{j=2}^{d}\left(t-\lambda_{j}\right)=t^{d-1}+b_{1} t^{d-2}+\cdots+b_{d-1}$ is a product of linear (for $\lambda_{j}$ real) and quadratic (for $\lambda_{j}, \bar{\lambda}_{j}$ complex) factors all of which have strictly positive coefficients. Hence $b_{j}>0,1 \leq j \leq d-1$. By the Cayley-Hamilton theorem, $\left(T^{d-1}+b_{1} T^{d-2}+\cdots+b_{d-1} I\right) x=0$, verifying the claim.

Now fix $z \in \mathbb{Z}^{d}$ with $\pi_{D}(z)>0$, and construct $A_{1, N}$ using $u_{1}=z, u_{2}=B z, \ldots, u_{d}=$ $B^{d-1} z$ as in the proof of theorem 1 with $M=1$. Since $B^{N} z$ is eventually in the positive cone of $\left\{u_{1}, \ldots, u_{d}\right\}$, it follows $A_{1, N} \geq 0$ for large enough $N$.

Suppose there were $C_{1}, \ldots, C_{n}$ with the property stated. Then for some $C_{j}$, infinitely many $\sigma_{A_{1, N}}$ factor onto $\sigma_{C_{j}}$. Pick $\delta$ so that $\operatorname{sp}\left(C_{j}\right) \subset\{|t|<(1-\delta) \lambda\} \cup\{\lambda\}$. There is an $N$ such that $\sigma_{A_{1, N}}$ factors onto $\sigma_{C_{j}}$ and, by the argument used in the cubic example, $\operatorname{sp}\left(A_{1, N}\right) \subset E(\lambda, 1, \delta)$. We now invoke a theorem of Kitchens [K] that if $\sigma_{A}$ factors onto $\sigma_{B}$, then $\chi_{B}(t)$ divides $\chi_{A}(t)$ modulo powers of $t$. Therefore suppose $\sigma_{A_{1, N}}^{k}$ factors onto $\sigma_{C_{i}}^{k}$ for some $k$. Since $\operatorname{sp}\left(A_{1, N}\right) \cap\{|t| \leq(1-\delta) \lambda\}=$ $\left\{\lambda_{2}, \ldots, \lambda_{d}\right\}$, Kitchens' result shows

$$
\prod_{\mu \in \operatorname{sp}\left(C_{i}\right)}\left(t-\mu^{k}\right) \mid \prod_{j=1}^{d}\left(t-\lambda_{j}^{k}\right) .
$$

Since $\operatorname{sp}\left(C_{i}\right) \supset\left\{\lambda_{1}, \ldots, \lambda_{d}\right\}$, the above shows $\operatorname{sp}\left(C_{i}\right)=\left\{\lambda_{1}, \ldots, \lambda_{d}\right\}$. Hence $\operatorname{tr} \lambda=$ $\operatorname{tr} C_{i} \geq 0$, contradicting the assumption on $\operatorname{tr} \lambda$.

The above argument shows that no assumption about the conjugates of $\lambda$ is needed to prove the first part of theorem 3. It appears likely that the whole theorem is true 
without such an assumption. For this to fail, there would have to exist $\lambda \in \mathbb{P}$ with $\operatorname{tr} \lambda<0$ simultaneously satisfying two conditions. The first is the existence of an aperiodic $C \geq 0$ of size $\leq(d-1) M_{0}(\lambda)+1=k$ such that for every eigenvalue of $C$ there is a $\lambda_{j}$ of equal modulus. Note there are only finitely many possible $\chi_{C}(t)$ 's to check, since the coefficients are integers bounded by $k ! \lambda^{k}$ and $\operatorname{deg} \chi_{C} \leq k$. The second condition is that the $(d-1) M$ eigenvalues in $\{|s|<(1-\delta) \lambda\}$ of all but finitely many of the $A_{M, N}$ constructed in the proof each have modulus the same as some $\lambda_{j}$. Numerical work suggests that neither of these possibilities occurs.

\section{REFERENCES}

[CP] E. M. Coven and M. E. Paul, Endomorphisms of irreducible subshifts of finite type, Math. Systems Th. 8 (1974), 167-175.

[DGS] M. Denker, C. Grillenberger, and K. Sigmund, Ergodic Theory on Compact Spaces, Springer Lecture Notes 527, New York, 1976.

[G] F. R. Gantmacher, The Theory of Matrices, v. II, Chelsea, New York, 1959.

[H] David Handelman, Positive matrices and dimension groups affiliated to $C^{*}$-algebras and topological Markov chains, J. Operator Theory 6 (1981), 55-74.

[K] Bruce Kitchens, An invariant for continuous factors of Markov shifts, Proc. Amer. Math. So. 83 (1981), 825-828.

[L] D. A. Lind, The entropies of topological Markov shifts and a related class of algebraic integers, Ergod. Th. and Dynam. Sys. 4 (1984), 283-300.

[M] B. Marcus, Factors and extensions of full shifts, Monats. Math. 88 (1979), 239-247.

[T] Paul Trow, Thesis, University of North Carolina, 1985.

[W] R. F. Williams, Classification of subshifts of finite type, Ann. of Math. 98 (1973), 120-153. 